\title{
On the minimal number of driving Lévy motions in a multivariate price model
}

\author{
Jean Jacod $^{*} \quad$ Mark Podolskij ${ }^{\dagger}$
}

May 20, 2017

\begin{abstract}
In this paper we consider the factor analysis for Lévy driven multivariate price models with stochastic volatility. Our main aim is to provide conditions on the volatility process under which we can possibly reduce the dimension of the driving Lévy motion. We will see that these conditions depend on a particular form of the multivariate Lévy process. In some settings we will concentrate on non-degenerate symmetric $\alpha$-stable Lévy motions.
\end{abstract}

Keywords: factor models, Lévy processes, semimartingales.

AMS 2010 Subject Classifications: 60H05, 60G51, 60G18.

\section{Introduction}

Multivariate Itô semimartingales are nowadays the commonly used models for multidimensional price processes in economics and finance. A lot of research has been devoted to understanding the dependence structure between the price components. The most prominent approaches include the factor models, which distinguish between the common and endogenous shocks, principal component analysis and the theory of cointegration. The common feature of these methods is the dimension reduction, which is extremely useful for practical calibration and simulation of the model.

The aim of this paper is to provide a simple factor analysis for multivariate Lévy driven price models that exhibit stochastic volatility. More specifically, we consider a filtered probability space $\left(\Omega, \mathcal{F},\left(\mathcal{F}_{t}\right)_{t \geq 0}, \mathbb{P}\right)$ satisfying the usual conditions and a $d$-dimensional process $X=\left(X^{j}\right)_{1 \leq j \leq d}$, which takes the form

$$
X_{t}=\int_{0}^{t} \sigma_{s} d Z_{s}
$$

where $Z$ is a symmetric Lévy motion with dimension $q$ and $\sigma$ is an $\mathbb{R}^{d \times q}$-valued predictable process (we often write $X=\sigma \cdot Z$ for notational simplicity). We would like to determine

\footnotetext{
*Institut de Mathématiques de Jussieu, Université Pierre et Marie Curie, 4 Place Jussieu, 75005 Paris, France, Email: jean.jacod@gmail.com.

${ }^{\dagger}$ Department of Mathematics, Aarhus University, Ny Munkegade 118, 8000 Aarhus, Denmark, Email: mpodolskij@math.au.dk.
} 
the minimal dimension of the driving Lévy motion such that the representation (1.1) holds. Mathematically speaking the central question of this paper is the following:

What is the smallest integer $r$ for which there exist two predictable processes $\gamma$ and $\sigma^{\prime}$ with values in $\mathbb{R}^{r \times q}$ and $\mathbb{R}^{d \times r}$ respectively, satisfying

$$
Z^{\prime}=\gamma \cdot Z \text { is a Lévy process, and } X=\sigma^{\prime} \cdot Z^{\prime} \text { ? }
$$

In relation to this problem we would also like to know whether the Lévy process $Z^{\prime}$ is of the same "type" as $Z$ (for example, does $Z^{\prime}$ have independent components if $Z$ does?). There are several examples in finance where the minimal dimension $r$ can be expected to be smaller than $d$. In [4] the authors mention the case of baskets of energy prices, where the required amount of driving Brownian motions is much smaller than $d$. We refer to [1, Chapter 5] for the detailed empirical analysis. Similar conclusions can be found in the LIBOR market models, where the principal component analysis suggests a sparse amount of Brownian motions that are accurately describing the dynamics; see [2, Section 6.19] for a more detailed discussion. Another example are bond prices with different maturities. In the situation of economic stability these prices have very similar trajectories and a rather minimal amount of driving Lévy motions might suffice to model the bonds. However, things can change drastically during an economic crisis.

When $Z$ and $Z^{\prime}$ are Brownian motions the formulated problem is indeed elementary. The answer is formulated in the theorem below. This statement can be found in [4] or, alternatively, it follows directly from the results of this paper, which will be presented later.

Theorem 1.1 Let $\overline{\mathbb{P}}$ denote the product measure $\mathbb{P}(d \omega) \otimes d t$ on $\left(\Omega \times \mathbb{R}_{+}, \mathcal{F} \otimes \mathcal{B}\left(\mathbb{R}_{+}\right)\right)$and assume that $Z$ is a standard d-dimensional Brownian motion. The minimal dimension $r$ for which (1.2) holds (and $Z^{\prime}$ is a $r$-dimensional Brownian motion) is the smallest integer such that the rank of the $\mathbb{R}^{d \times q}$-valued matrix $\sigma(\omega)_{t}$ is not larger than $r$, outside a $\overline{\mathbb{P}}$-null set of $\Omega \times \mathbb{R}_{+}$.

This characterization has been used in $[3,4,5]$ to construct statistical tests for the minimal dimensional of the Brownian motion in the setting of continuous diffusion models possibly contaminated by microstructure noise. As we will see later, the result of Theorem (1.1) extends to all isotropic Lévy processes $Z$ (meaning that $\Pi Z$ has the same law as $Z$ for any orthogonal $q \times q$ matrix $\Pi$ ). Otherwise, except under some very special conditions, typically on both $\sigma$ and $Z$, the minimal $r$ for which (1.2) holds is $r=q$, and no dimension reduction is available. This is probably not surprising when $Z$ is an arbitrary Lévy process without any special structure. A more surprising analysis appears in what we call the i.i.d. case, where all components $Z^{i}$ have the same law and are independent. We will study this setting in more details and characterize the minimal $r$ in (1.2) when the process $Z^{1}$ is symmetric $\alpha$-stable for any $d$, and when $Z^{1}$ is an arbitrary symmetric Lévy process and $d=1$. Even in these cases the characterization is indeed somewhat involved.

The paper is structured as follows. In Section 2 we present some basics from the theory of Itô semimartingales and Lévy processes. Section 3 is devoted to the study of the isotropic setting, where the obtained result is similar to Theorem 1.1. We investigate the one-dimensional case, which turns out to be not quite trivial, in Section 4. Finally, in Section 5 we give a complete answer to the problem (1.2) in the framework of the i.i.d. stable motion $Z$. 


\section{Preliminaries}

In this section we introduce some notation and recall some basic facts from the theory of semimartingales and Lévy processes.

We denote by $\mathcal{M}_{r, q}$ the vector space of $r \times q$ matrices. For any $A \in \mathcal{M}_{r, q}$, we write $A^{\star} \in \mathcal{M}_{q, r}$ for the transpose of $A$. The space of all orthogonal $\mathbb{R}^{q \times q}$-valued matrices is denoted by $\mathcal{P}_{q}$. For any $q \geq 1$, we write $S_{q-1}$ for the unit sphere in $\mathbb{R}^{q}$.

First of all, we recall that a $q$-dimensional Lévy process $Z$ is uniquely characterized by the Lévy triplet $(b, c, F)$, where $b$ is an $\mathbb{R}^{q}$-valued drift, $c$ is a positive semidefinite volatility matrix in $\mathcal{M}_{q, q}$ and $F$ is a measure on $\mathbb{R}^{q}$ satisfying $\int_{\mathbb{R}^{q}} \min \left(1,\|x\|^{2}\right) F(d x)<\infty$. In particular, the characteristic function of $Z_{t}$ is given by $\mathbb{E}\left[\exp \left(i u^{\star} Z_{t}\right)\right]=\exp \left(t \phi^{Z}(u)\right)$, where the characteristic exponent $\phi^{Z}$ has the representation

$$
\phi^{Z}(u)=i u^{\star} b-\frac{1}{2} u^{\star} c u+\int_{\mathbb{R}^{q}}\left(\exp \left(i u^{\star} x\right)-1-i u^{\star} x 1_{\{\|x\| \leq 1\}}\right) F(d x) .
$$

We refer to the book [6] for a detailed exposition of Lévy processes. A particular class of Lévy processes are the non-degenerate $q$-dimensional symmetric $\alpha$-stable processes $(\alpha \in(0,2))$ on $\left(\Omega, \mathcal{F},\left(\mathcal{F}_{t}\right)_{t \geq 0}, \mathbb{P}\right)$, which we denote by $S S S_{q}^{\alpha}$. We recall that a $q$-dimensional Lévy process is in $S S S_{q}^{\alpha}$ if and only if its characteristic triple is $(0,0, F)$, with the Lévy measure factorizing as

$$
F(d x)=\frac{1}{C_{\alpha} \rho^{1+\alpha}} d \rho H(d \theta) \quad \text { with } \quad C_{\alpha}=\int_{0}^{\infty} \frac{1-\cos x}{x^{1+\alpha}} d x,
$$

where $x=(\rho, \theta) \in \mathbb{R}_{+} \times S_{q-1}$ and $H$ is a finite positive measure on the unit sphere $S_{q-1}$ of $\mathbb{R}^{q}$ (the directional measure), which is symmetric (i.e. invariant by the map $\theta \mapsto-\theta$ ) and satisfies $H\left(\left\{\theta: \lambda^{\star} \theta \neq 0\right\}\right)>0$ for all $\lambda \in S_{q-1}$ (the latter reflects the non-degeneracy of the process). In this setting the characteristic exponent of $Z$ takes the following simple form

$$
\phi^{Z}(u)=-\int_{S_{q-1}}\left|u^{\star} \theta\right|^{\alpha} H(d \theta) .
$$

We notice that the process $Z \in S S S_{q}^{\alpha}$ is isotropic if and only if $H$ is proportional to the Lebesgue measure on $S_{q-1}$, or equivalently if and only if $\phi^{Z}(u)=-a\|u\|^{\alpha}$ for some constant $a>0$. On the other hand, $Z \in S S S_{q}^{\alpha}$ has i.i.d. components if and only if $H=\frac{a}{2} \sum_{i=1}^{q}\left(\varepsilon_{e_{i}}+\varepsilon_{-e_{i}}\right)$ where $\left(e_{i}\right)_{1 \leq i \leq q}$ is the canonical basis of $\mathbb{R}^{q}, \varepsilon_{x}$ denotes the Dirac measure at $x$ and $a>0$, or equivalently if and only if $\phi^{Z}(u)=a \sum_{i=1}^{q}\left|u^{i}\right|^{\alpha}$. We remark that these two types of $S S S_{q}^{\alpha}$-processes are different when $\alpha \in(0,2)$, but they coincide for $\alpha=2$ which corresponds to the case of Brownian motion.

Before we start with the preliminary analysis we make the following simple observation. The "true" dimension of the driving Lévy motion $Z$ in (1.1) may be very well smaller than $q$. Indeed, there is a minimal linear subspace $V$ of $\mathbb{R}^{q}$ in which $Z$ really lives, which is the linear subspace spanned by the support of the law of $Z_{t}$ (it is the same for all $t>0$ due to the properties of a Lévy process). Let $q^{\prime}$ be the dimension of $V$. Then if $q^{\prime}<q$ the true dimension of $Z$ is $q^{\prime}$ in the following sense: there is a $\Pi \in \mathcal{P}_{q}$ such that $\Pi x$ belongs to the subspace spanned by the first $q^{\prime}$ elements of the canonical basis of $\mathbb{R}^{q}$, for all $x \in H$. Then $\bar{Z}=\Pi Z$ is a Lévy process with components $\bar{Z}^{i}$ for $i>q^{\prime}$ being identically vanishing, and if $Z^{\prime}=\left(\bar{Z}^{1}, \ldots, \bar{Z}^{q^{\prime}}\right)^{\star}$, (1.1) implies $X=\sigma^{\prime} \cdot Z^{\prime}$ for the process $\sigma_{t}^{\prime}$ which is the $d \times q^{\prime}$ 
left block of the matrix $\sigma_{t} \Pi^{\star}$. It is of course natural to do this trivial dimension reduction before starting to solve the original problem. Hence, it is no restriction to assume that $Z$ is non-degenerate in the sense that the linear subspace generated by the support of $Z_{1}$ is $\mathbb{R}^{q}$.

Next, we recall some well known facts about Itô semimartingales. A $d$-dimensional semimartingale $Y$ on $\left(\Omega, \mathcal{F},\left(\mathcal{F}_{t}\right)_{t \geq 0}, \mathbb{P}\right)$ is called an Itô semimartingale if its characteristics are absolutely continuous (in time) with respect to Lebesgue measure, and their derivatives with respect to time are called the spot characteristics. More specifically, they consist of a spot triple $\left(b_{t}, c_{t}, F_{t}\right)$ where $b_{t}$ is the drift (a $d$-dimensional predictable process), $c_{t}$ is the diffusion coefficient (predictable with values in the set of symmetric nonnegative definite $d \times d$ matrices) and $F_{t}$ is the spot Lévy measure on $\mathbb{R}^{d}$ (predictable with $\int_{\mathbb{R}^{d}} \min \left(1,\|x\|^{2}\right) F_{t}(d x)<$ $\infty)$. The "spot characteristic exponent" at time $t$ is the function $\phi_{t}=\phi_{\omega, t}$ on $\mathbb{R}^{d}$ defined by

$$
\phi_{t}^{Y}(u)=i u^{\star} b_{t}-\frac{1}{2} u^{\star} c_{t} u+\int_{\mathbb{R}^{p}}\left(\exp \left(i u^{\star} x\right)-1-i u^{\star} x 1_{\{\|x\| \leq 1\}}\right) F_{t}(d x),
$$

and it characterizes $\left(b_{t}, c_{t}, F_{t}\right)$ for any $(\omega, t)$. In fact, $\phi_{t}^{Y}(u)$ is uniquely determined up to a

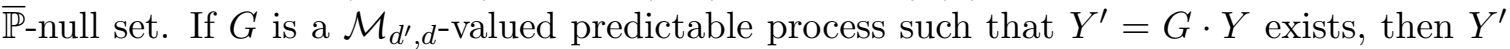
is also an Itô semimartingale and

$$
\phi_{t}^{Y^{\prime}}(u)=\phi_{t}^{Y}\left(G_{t}^{\star} u\right) \quad \forall u \in \mathbb{R}^{d^{\prime}} .
$$

Moreover, $Y$ is Lévy if and only if there exists a version of $\phi_{t}^{Y}$ (equivalently, of $\left(b_{t}, c_{t}, F_{t}\right)$ ) which is independent of $(\omega, t)$. Then $\phi^{Y}(u)$ is the characteristic exponent and $(b, c, F)$ is the characteristic triplet. The Lévy process $Y$ is non-degenerate if and only if $\phi^{Y}(u) \neq 0$ for all non-vanishing $u \in \mathbb{R}^{d}$, and symmetric if an only if $\phi^{Y}(u)=\phi^{Y}(-u)$.

We are now ready to give a necessary and sufficient condition for having (1.2) (recall that an integral process $\psi \cdot Z$ is unchanged if we modify $\psi$ on a $\overline{\mathbb{P}}$-null set).

Lemma 2.1 Consider the model (1.1) driven by a non-degenerate Lévy process $Z$. Let $\gamma$ and $\sigma^{\prime}$ be predictable processes with values in $\mathcal{M}_{r, q}$ and $\mathcal{M}_{d, r}$, respectively. Then (1.2) holds if and only if, outside an $\overline{\mathbb{P}}$-null set, we have:

$$
\left.\begin{array}{l}
\sigma(\omega)_{t}=\sigma^{\prime}(\omega)_{t} \gamma(\omega)_{t} \\
\phi^{Z}\left(\gamma(\omega)_{t}^{*} v\right) \quad \text { is independent of }(\omega, t) \text { for all } v \in \mathbb{R}^{r}
\end{array}\right\}
$$

Proof. The second condition of (2.7) implies that the stochastic integral process $Z^{\prime}=\gamma \cdot Z$ is well defined, and it is a Lévy process with characteristic exponent $\phi^{Z^{\prime}}(u)=\phi^{Z}\left(\Gamma^{\star} u\right)$ where $\Gamma=\gamma(\omega)_{t}$ for any $(\omega, t)$ outside the above-mentioned $\overline{\mathbb{P}}$-null set. Then the first condition of (2.7) implies $\sigma^{\prime} \cdot Z^{\prime}=\left(\sigma^{\prime} \gamma\right) \cdot Z=X$ and (1.2) holds.

Conversely, assume (1.2). Clearly, the second part of (2.7) holds. As for the first part, observe that a combination of (1.1) and (1.2) yields $G \cdot Z=0$, where $G_{t}=\sigma_{t}-\sigma_{t}^{\prime} \gamma_{t}$. Then we must have $\phi_{t}^{G \cdot Z}(u)=0$, hence $\phi^{Z}\left(G_{t}^{\star} u\right)=0$ outside a $\overline{\mathbb{P}}$-null set and for all $u \in \mathbb{R}^{d}$. Since $Z$ is non-degenerate, this implies $G_{t}^{\star} u=0$ for all $u$. Hence $G_{t}=0$, and the proof is complete.

When the first part of (2.7) holds, the rank of $\sigma(\omega)_{t}$ is not more than $r$. Then a simple consequence of the previous lemma is the following result: 
Corollary 2.2 Assume (1.1) with $Z$ being a non-degenerate Lévy process. If (1.2) holds for some integer $r$, then outside a $\overline{\mathbb{P}}$-null set the rank of $\sigma(\omega)_{t}$ is not bigger than $r$.

On the other hand, if the rank of $\sigma(\omega)_{t}$ is not bigger than $r$, one can find $\sigma^{\prime}(\omega)_{t} \in \mathcal{M}_{d, r}$ and $\gamma(\omega)_{t} \in \mathcal{M}_{r, q}$ such that the first part of (2.7) holds (see for example the proof of Theorem 3.1 below), and when $\operatorname{rank}\left(\sigma(\omega)_{t}\right) \leq r$ for all $(\omega, t)$ it is no problem to find predictable versions for $\sigma_{t}^{\prime}$ and $\gamma_{t}$. However, there is no reason why the second part of (2.7) should hold, except of course when $\sigma(\omega)_{t}$, hence $\sigma^{\prime}(\omega)_{t} \in \mathcal{M}_{d, r}$ and $\gamma(\omega)_{t} \in \mathcal{M}_{r, q}$ as well, are independent of $(\omega, t)$. So we have:

Corollary 2.3 Assume (1.1) with $Z$ being a non-degenerate Lévy process, and $\sigma(\omega)_{t}=\sigma$ a constant matrix. The minimal integer $r$ for which (1.2) holds is the rank $m$ of $\sigma$.

Unfortunately, for the reason mentioned above, this result (with $m$ the $\overline{\mathbb{P}}$-essential supremum of $\left.\operatorname{rank}\left(\sigma(\omega)_{t}\right)\right)$ fails in general, except in the isotropic case.

\section{The isotropic case}

In this section we treat the isotropic case. As mentioned earlier, when $Z$ is a non-degenerate isotropic Lévy process, the result of Theorem 1.1 remains valid.

Theorem 3.1 We consider the model (1.1) with $Z$ being a non-degenerate isotropic Lévy process. The minimal dimension $r$ for which (1.2) holds is the smallest integer such that the rank of the matrix $\sigma(\omega)_{t}$ is not larger than $r$ outside an $\overline{\mathbb{P}}$-null set. Furthermore, we can choose $Z^{\prime}$ in (1.2) to be isotropic as well.

Proof. Let $m(\omega)_{t}=\operatorname{rank}\left(\sigma(\omega)_{t}\right)$. Up to a modification on a $\overline{\mathbb{P}}$-null set, we can assume that there exists a $m \in \mathbb{N}$ such that

$$
m_{t} \leq m \quad \text { and } \quad m_{t}=m \text { on a set } A \text { with } \overline{\mathbb{P}}(A)>0 .
$$

Omitting the dependence on $\omega$, we can decompose $\sigma_{t}$ as $\sigma_{t}=\Pi_{t} \Lambda_{t} \Pi_{t}^{\prime}$ where $\Pi_{t}, \Pi_{t}^{\prime}, \Lambda_{t}$ are predictable processes with respective values in $\mathcal{P}_{d}, \mathcal{P}_{q}, \mathcal{M}_{d, q}$, with all entries of $\Lambda_{t}$ equal to 0 except possible for $\lambda_{t}^{i}=\Lambda_{t}^{i i}$ when $i \leq m$. We also consider the $\mathcal{M}_{d, m}$-valued process $\Lambda_{t}^{\prime}$ consisting in the upper left $d \times m$ block of $\Lambda_{t}$.

The isotropy of $Z$ implies the identity $\phi^{Z}\left(\Pi^{\prime \prime} u\right)=\phi^{Z}(u)$ for any $u \in \mathbb{R}^{q}$ and $\Pi^{\prime \prime} \in \mathcal{P}_{q}$. Then $\phi^{Z}\left(\Pi_{t}^{\prime} u\right)=\phi^{Z}(u)$, which shows by $(2.6)$ that the process $\bar{Z}=\Pi^{\prime} \cdot Z$ is well defined and has the same law as $Z$ itself. Thus the $m$-dimensional process $Z^{\prime}$ whose components are the first $m$ components of $\bar{Z}$ is obviously an isotropic Lévy process. Moreover, we have

$$
X=\sigma \cdot Z=\left(\Pi \Lambda \Pi^{\prime}\right) \cdot Z=(\Pi \Lambda) \cdot \bar{Z}=\left(\Pi \Lambda^{\prime}\right) \cdot Z^{\prime},
$$

where the last equality follows from $\Lambda_{t}^{i j}=0$ when $j>m$. Hence (1.2) holds with $r=m$ and $\sigma_{t}^{\prime}=\Pi_{t} \Lambda_{t}^{\prime}$.

Conversely, assume (1.2) for some $r$. Then (2.7) implies the identity $\sigma_{t}=\sigma_{t}^{\prime} \gamma_{t}$. This yields $m_{t} \leq r$, and hence $m \leq r$ as well. 
The characterization of Theorem 3.1 was the basis for statistical tests for the minimal number of Brownian motions in continuous diffusion models that have been developed in $[3,5]$. In these papers the authors employed the following matrix perturbation method to uncover the rank $r$ of a positive semi-definite matrix $A \in \mathcal{M}_{d, d}$. For an arbitrary positive definite matrix $B \in \mathcal{M}_{d, d}$, the multi-linearity of the determinant implies the asymptotic expansion

$$
\operatorname{det}(A+\lambda B)=\lambda^{d-r} \gamma_{r}(A, B)+O\left(\lambda^{d-r+1}\right) \quad \text { as } \quad \lambda \downarrow 0,
$$

where the constant $\gamma_{r}(A, B)$ is given by $\gamma_{r}(A, B)=\sum_{C \in \mathbb{M}_{A, B}^{r}} \operatorname{det}(C)$ and the set $\mathbb{M}_{A, B}^{r}$ is defined via

$$
\mathbb{M}_{A, B}^{r}:=\left\{C \in \mathcal{M}_{d, d}: C_{i}=A_{i} \text { or } C_{i}=B_{i} \text { with } \#\left\{i: C_{i}=A_{i}\right\}=r\right\} .
$$

This expansion is the key to identification of the unknown rank $r$ of the matrix $A$. Indeed, when $\gamma_{r}(A, B) \neq 0$ we deduce the convergence

$$
\frac{\operatorname{det}(A+2 \lambda B)}{\operatorname{det}(A+\lambda B)} \rightarrow 2^{d-r} \quad \text { as } \quad \lambda \downarrow 0 .
$$

In [5] the latter idea has been applied to random perturbation of high frequency observations of the process $X$ driven by a $d$-dimensional Brownian motion $Z$ to perform hypothesis testing for the maximal rank of the squared volatility process $c_{t}=\sigma_{t} \sigma_{t}^{\star}$, hence deriving statistical methods to answer the question formulated in (1.2). Later on this approach has been extended to continuous diffusion models observed with microstructure noise in [3]. However, due to substantial differences in the asymptotic theory, the extension of this idea to general isotropic Lévy processes seems to be out of reach.

\section{The one-dimensional case}

While in the isotropic case the question at (1.2) is fully answered in Theorem 3.1, things are less obvious if we drop this isotropy condition. In this section we explicitly concentrate on the case $d=1$, but keep $q \geq 2$. Hence, the process $\sigma^{\star}$ at (1.1) becomes $\mathbb{R}^{q}$-valued. Our first result is treating the stable case.

Theorem 4.1 We consider the model (1.1) with $d=1$ and $Z \in S S S_{q}^{\alpha}$. Then (1.2) holds with $r=1$ and we can choose $Z^{\prime}$ in $S S S_{1}^{\alpha}$.

Proof. Let $e_{1}=(1,0, \ldots, 0)^{\star}$ be the first element of the canonical basis of $\mathbb{R}^{q}$. Recalling the representation (2.4), we see that both $a=\phi^{Z}\left(e_{1}^{\star}\right)$ and $\phi^{Z}\left(\sigma_{t}^{\star}\right)$ are negative. We define the predictable processes

$$
\sigma_{t}^{\prime}=\left(-\phi^{Z}\left(\sigma_{t}^{\star}\right)\right)^{1 / \alpha}, \quad \gamma_{t}=\left(\frac{1}{\sigma_{t}^{\prime}} 1_{\left\{\sigma_{t}^{\prime}>0\right\}}\right) \sigma_{t}+\left(\frac{1}{(-a)^{1 / \alpha}} 1_{\left\{\sigma_{t}^{\prime}=0\right\}}\right) e_{1}^{\star} .
$$

Note that $\sigma_{t}^{\prime} \geq 0$ by (2.4), and $\sigma_{t}^{\prime}=0 \Leftrightarrow \sigma_{t}=0$. We obviously have $\sigma_{t}=\sigma_{t}^{\prime} \gamma_{t}$, and for $v \in \mathbb{R}$ we deduce

$\sigma_{t} \neq 0 \Rightarrow \phi^{Z}\left(\gamma_{t}^{\star} v\right)=\phi^{Z}\left(\frac{v}{\sigma_{t}^{\prime}} \sigma_{t}^{\star}\right)=-|v|^{\alpha}, \quad \sigma_{t}=0 \Rightarrow \phi^{Z}\left(\gamma_{t}^{\star} v\right)=\phi^{Z}\left(\frac{v}{a^{1 / \alpha}} e_{1}\right)=-|v|^{\alpha}$. 
Hence, (2.3) is satisfied and by Lemma 2.1 we obtain (1.2) with $Z^{\prime}=\gamma \cdot Z$ which satisfies $\phi^{Z^{\prime}}(v)=-|v|^{\alpha}$. Thus $Z^{\prime} \in S S S_{1}^{\alpha}$ and the proof is complete.

When $Z$ is a Lévy process the same result does not hold in general, but we have a criterion under which it holds when further $Z$ is a non-degenerate symmetric Lévy process without a Gaussian part. We need to introduce some additional notation: for any measure $\mu$ on $\mathbb{R}^{p}$ and any $y \in \mathbb{R}^{p}$ we denote by $\mu^{(y)}$ the measure on $\mathbb{R}$, which is the image of $\mu$ by the map $x \mapsto y^{\star} x$.

Theorem 4.2 We consider the model (1.1) with $d=1$ and $Z$ being a q-dimensional nondegenerate symmetric Lévy process without a Gaussian part. Then (1.2) holds with $r=1$ if and only if there exist a measure $\mu$ on $\mathbb{R}$ with $\mu(\{0\})=0$ and a nonnegative predictable process $a_{t}$ such that, outside of a $\overline{\mathbb{P}}$-null set, we have

$$
F^{\left(\sigma_{t}^{\star}\right)}=\mu^{\left(a_{t}\right)},
$$

where $F$ is the Lévy measure of $Z$.

Proof. The non-degeneracy of $Z$ and $F(\{0\})=0$ imply that $F^{(y)}=0$ holds if and only if $y=0$. We have two cases: either $\sigma_{t}=0 \widehat{\mathbb{P}}$-a.s. and (1.2) holds with $\sigma^{\prime} \equiv 0$ and, say $Z^{\prime}=Z^{1}$ and (4.8) holds with $\mu=0$; or $\overline{\mathbb{P}}(\{\sigma \neq 0\})>0$ and if (4.8) holds the measure $\mu$ is non-vanishing. In the sequel we discard the first trivial case.

a) Here we prove that (4.8) implies the representation (1.2) with $r=1$. Upon a modification of the process $\sigma$ on an $\overline{\mathbb{P}}$-null set we may assume (4.8) to hold for all $(\omega, t)$. We pick a vector $\delta \in \mathbb{R}^{q}$ such that $F^{(\delta)}=\mu$ (such a vector exists by (4.8)) and set

$$
\gamma_{t}=\frac{1}{a_{t}} \sigma_{t} 1_{\left\{a_{t}>0\right\}}+\delta^{\star} 1_{\left\{a_{t}=0\right\}}, \quad \sigma_{t}^{\prime}=a_{t} .
$$

Then, for $v \in \mathbb{R}$, we obtain the identities

$$
\begin{aligned}
\phi^{Z}\left(v \gamma_{t}^{\star}\right) & =\int_{\mathbb{R}^{q}}\left(\exp \left(i v \gamma_{t} x\right)-1\right) F(d x) \\
& =1_{\left\{a_{t}>0\right\}} \int_{\mathbb{R}^{q}}\left(\exp \left(i v \sigma_{t} x / a_{t}\right)-1\right) F(d x)+1_{\left\{a_{t}=0\right\}} \int_{\mathbb{R}^{q}}(\exp (i v \delta x)-1) F(d x) \\
& =1_{\left\{a_{t}>0\right\}} \int_{\mathbb{R}}\left(\exp \left(i v z / a_{t}\right)-1\right) F^{\left(\sigma_{t}^{\star}\right)}(d z)+1_{\left\{a_{t}=0\right\}} \int_{\mathbb{R}}(\exp (i v z)-1) F^{(\delta)}(d z) \\
& =1_{\left\{a_{t}>0\right\}} \int_{\mathbb{R}}(\exp (i v z)-1) \mu(d z)+1_{\left\{a_{t}=0\right\}} \int_{\mathbb{R}}(\exp (i v z)-1) \mu(d z) \\
& =\int_{\mathbb{R}}(\exp (i v z)-1) \mu(d z) .
\end{aligned}
$$

This implies the second part of (2.7). Moreover, $\mu^{(0)}=0$ because $\mu(\{0\})=0$. So, if $a_{t}=0$ we have $F^{\left(\sigma_{t}^{\star}\right)}=0$, which as mentioned earlier implies $\sigma_{t}=0$. Therefore the first part of (2.7) is obvious, and (1.2) holds with $r=1$ holds.

b) Conversely, assume that (1.2) holds with $r=1$. Hence, we can assume that (2.7) holds identically for some predictable processes $\gamma, \sigma^{\prime}$, and $Z^{\prime}=\gamma \cdot Z$. Let $\mu$ be the Lévy measure of $Z^{\prime}$. Then $X=\sigma \cdot Z=\sigma^{\prime} \cdot Z^{\prime}$ implies that the spot Lévy measure of $X$ is $F_{t}^{X}=F^{\left(\sigma_{t}^{\star}\right)}$ and also $F_{t}^{X}=\mu^{\left(\sigma_{t}^{\prime}\right)}$. Hence, (4.8) holds with this $\mu$ and $a_{t}=\sigma_{t}^{\prime}$. 
When $Z$ is isotropic, so is $F$, and it is simple to heck (4.8) for any process $\sigma_{t}$ : one may take $\mu=F^{\left(e_{1}\right)}$ and $a_{t}=\left\|\sigma_{t}\right\|$. When $Z \in S S S_{q}^{\alpha}$ (4.8) again holds for any process $\sigma_{t}$ : we take for $\mu$ the Lévy measure of a one-dimensional symmetric $\alpha$-stable process, and $F^{(y)}$ is necessarily of the same type for any $y \in \mathbb{R}^{q} \backslash\{0\}$.

One may then wonder whether, for a given $Z$ as in the previous theorem, (4.8) holds for all $\mathcal{M}_{1, q}$-valued process $\sigma$ (with $a_{t}$ depending on $\sigma_{t}$, of course). We do not know a criterion for this question except in the i.i.d. case.

Theorem 4.3 Let $Z$ be an i.i.d. symmetric non-degenerate Lévy process without a Gaussian part. Then (1.1) with $d=1$ implies (1.2) with $r=1$ for any predictable $\mathcal{M}_{1, q}$-valued process $\sigma_{t}$ if and only if $Z \in S S S_{q}^{\alpha}$.

Proof. The sufficient condition follows from the Theorem 4.1. For the necessary condition, we denote by $\nu$ the Lévy measure of all components of $Z$ and $\left(e_{j}\right)$ the canonical basis of $\mathbb{R}^{q}$. Then $F(A)=\sum_{j=1}^{d} \int_{\mathbb{R}} 1_{A}\left(z e_{j}\right) \nu(d z)$ for $A \subset \mathbb{R}^{q}$, and hence

$$
F^{\left(\sigma_{t}^{\star}\right)}=\sum_{j=1}^{q} \nu^{\left(\sigma_{t}^{j}\right)}
$$

If we take the process with components $\sigma_{t}^{1}=1$ and $\sigma_{t}^{2}=1_{\{t>1\}}$ and $\sigma_{t}^{j}=0$ for $j \geq 2$, Theorem 4.2 yields a measure $\mu$ on $\mathbb{R}$ and a nonnegative process $a_{t}$ such that

$$
t \leq 1 \Rightarrow \nu=\mu^{\left(a_{t}\right)}, \quad t>1 \Rightarrow 2 \nu=\mu^{\left(a_{t}\right)} .
$$

Then we can take $\mu=\nu$ and $a_{t}=1$ for $t \leq 1$, and the second part above yields $2 \nu=\nu^{(b)}$ for some $b>0$ and all $s \geq 0$. If $\bar{\nu}(x)=\nu((x, \infty))$ is the tail of the symmetric measure $\nu$, we thus have $2 \bar{\nu}(x)=\bar{\nu}(x / b)$ for all $x>0$, which implies that $\bar{\nu}$ has the form $\bar{\nu}(x)=C x^{-\alpha}$ for some constants $C$, $\alpha$, with necessarily $\alpha \in(0,2)$ because $\bar{\nu}$ is the tail of a Lévy measure. This implies that indeed the components $Z^{j}$ are symmetric $\alpha$-stable.

\section{The stable i.i.d. case}

In this last section we suppose that $d \geq 2$. In view of the previous results for the general Lévy motions, finding the minimal $r$ in (1.2) seems out of reach. Hence, we assume here that $Z \in S S S_{q}^{\alpha}$. As a matter of fact, in the general non-isotropic stable setting, the problem also seems very difficult to analyze, except in the i.i.d. case. So, we restrict our attention to this framework.

The interpretation of the minimal $r$ for which (1.2) holds as the number of factors is clearer if we ask $Z^{\prime}$ to be i.i.d. as well, and we begin with this case. We need some additional notation. Omitting $\omega$, we introduce for $j=0, \ldots, q$ the predictable integer-valued processes $M_{t}^{j}$ by induction on $j\left(\sigma_{t}^{\cdot, j}\right.$ below denotes the $j$ th column vector of the matrix $\left.\sigma_{t}\right)$ :

$M_{t}^{0}=0, \quad M_{t}^{j+1}= \begin{cases}M_{t}^{j} & \text { if } \sigma_{t}^{\cdot, j+1} \text { vanishes, or is proportional to } \sigma_{t}^{\cdot, l} \text { for some } 1 \leq l \leq j \\ M_{t}^{j}+1 & \text { otherwise. }\end{cases}$ 
Then $m_{t}=M_{t}^{q}$ is the number of column vectors of the matrix $\sigma_{t}$, which are pairwise noncollinear (with the convention $m_{t}=0$ when $\sigma_{t}=0$ ). If $r_{t}$ is the rank of $\sigma_{t}$, we have $m_{t}=r_{t}$ if $r_{t}=0,1$, and if $r_{t} \geq 2$ then $m_{t}$ can attain all values between $r_{t}$ and $q$.

Theorem 5.1 We consider the model (1.1) with $Z$ being an i.i.d. symmetric $\alpha$-stable motion. The minimal dimension $r$ for which (1.2) holds with $Z^{\prime}$ being i.i.d. symmetric $\alpha$-stable motion is the smallest integer $m$ such that $m(\omega)_{t} \leq m$ outside an $\overline{\mathbb{P}}$-null set.

Proof. As in the proof of Theorem 3.1 we may assume that $m_{t} \leq m$ identically, and $m(\omega)_{t}=m$ on a subset $A$ with $\overline{\mathbb{P}}(A)>0$. We also assume $m<q$; otherwise there is nothing to prove.

a) In order to simplify the analysis, we perform a kind of permutation of the coordinates of $Z$, according to a random and time dependent scheme. Let $I=\{1, \ldots, q\}$. With the notation (5.9), we denote by $J_{t}^{1}, \cdots, J_{t}^{m_{t}}$ the ordered indices $j$ in $I$ such that $M_{t}^{j}=M_{t}^{j-1}+1$, and by $J_{t}^{m_{t}+1}, \ldots, J_{t}^{q}$ the ordered indices $j$ in $I$ such that $M_{t}^{j}=M_{t}^{j-1}$, so $j \mapsto J_{t}^{j}$ is a bijection from $I$ onto itself, and its inverse is denoted by $j \mapsto N_{t}^{j}$ (so $J_{t}^{N_{t}^{j}}=j$ ). Note that $J_{t}^{j}$ and $N_{t}^{j}$ are predictable, as well as the invertible matrix $\zeta_{t}^{i j}=1_{\left\{j=J_{t}^{i}\right\}}=1_{\left\{i=N_{t}^{j}\right\}}$. So the $q$-dimensional process $\bar{Z}=\zeta \cdot Z$ is well defined and, since for $u=\left(u_{i}\right)_{i \in I} \in \mathbb{R}^{q}$ the components of $\zeta_{t}^{\star} u$ are $u_{N_{t}^{i}}$, its spot characteristic exponent is

$$
\phi_{t}^{\bar{Z}}(u)=\phi^{Z}\left(\zeta_{t}^{\star} u\right)=-a \sum_{i=1}^{q}\left|\left(\zeta_{t}^{\star} u\right)_{i}\right|^{\alpha}=-a \sum_{i=1}^{q}\left|u_{N_{t}^{i}}\right|^{\alpha}=-a \sum_{i=1}^{q}\left|u_{i}\right|^{\alpha}
$$

for some $a>0$. Therefore, $\bar{Z}$ is an i.i.d. symmetric $\alpha$-stable Lévy motion. Moreover, for any predictable $\mathcal{M}_{r, q^{-}}$valued process $\psi_{t}$, we have $\psi \cdot Z=\left(\psi_{t} \zeta_{t}^{-1}\right) \cdot \bar{Z}$. So, upon replacing $\sigma_{t}$ by $\bar{\sigma}_{t}=\sigma_{t} \zeta^{-1}$, which amounts to do for each $t$ a permutation of the columns (hence letting the number $m_{t}$ unchanged), it is enough to prove the result for $X=\bar{\sigma} \cdot \bar{Z}$.

Equivalently, this amounts to solve the original problem under the additional assumption that $J_{t}^{j}=j$ identically for all $j \in I$. In other words, the columns $\sigma_{t}^{, j}$ for $j=1, \ldots, m_{t}$ are pairwise non collinear, and each other column vector is a multiple of one of the first $m_{t}$ columns vectors. Hence, since $m_{t} \leq m$, for $j=m+1, \ldots, q$ there are predictable processes $\beta_{t}^{j}$ and $L_{t}^{j}$ with values in $\mathbb{R}$ and $\{1, \ldots, m\}$ such that

$$
j>m \Rightarrow \sigma_{t}^{\cdot, j}=\beta_{t}^{j} \sigma_{t}^{\cdot L_{t}^{j}} .
$$

b) We define the predictable processes $\rho_{t}^{i}$ and $\gamma_{t}^{i j}$ for $1 \leq i \leq m$ and $j \in I$ by

$$
\rho_{t}^{i}=1+\sum_{l=m+1}^{q}\left|\beta_{t}^{l}\right|^{\alpha} 1_{\left\{L_{t}^{l}=i\right\}}, \quad \gamma_{t}^{i j}=\frac{1}{\left(\rho_{t}^{i}\right)^{1 / \alpha}}\left(1_{\{i=j \leq m\}}+\beta_{t}^{j} 1_{\left\{L_{t}^{j}=i, j>m\right\}}\right) .
$$

Then, for any $u=\left(u_{i}\right) \in \mathbb{R}^{m}$, we see that

$$
\begin{aligned}
\sum_{j=1}^{q}\left|\left(\gamma_{t}^{\star} u\right)_{j}\right|^{\alpha} & =\sum_{j=1}^{q}\left|\sum_{i=1}^{m} \frac{u_{i}}{\left(\rho_{t}^{i}\right)^{1 / \alpha}}\left(1_{\{i=j \leq m\}}+\beta_{t}^{j} 1_{\left\{L_{t}^{j}=i, j>m\right\}}\right)\right|^{\alpha} \\
& =\sum_{j=1}^{m} \frac{\left|u_{j}\right|^{\alpha}}{\rho_{t}^{j}}+\sum_{j=m+1}^{q}\left|\beta_{t}^{j}\right|^{\alpha} \frac{\left|u_{L_{t}^{j}}\right|^{\alpha}}{\rho_{t}^{j}} \\
& =\sum_{i=1}^{m} \frac{\left|u_{i}\right|^{\alpha}}{\rho_{t}^{i}}\left(1+\sum_{j=m+1}^{q}\left|\beta_{t}^{j}\right|^{\alpha} 1_{\left\{L_{t}^{j}=i\right\}}\right)=\sum_{i=1}^{m}\left|u_{i}\right|^{\alpha},
\end{aligned}
$$


from which we deduce $\phi^{Z}\left(\gamma_{t}^{\star} u\right)=-a \sum_{i=1}^{m}\left|u_{i}\right|^{\alpha}$. Thus, $\gamma$ is integrable with respect to $Z$, and $Z^{\prime}=\gamma \cdot Z$ is an i.i.d. symmetric $\alpha$-stable Lévy motion. A simple computation shows that $\sigma_{t}=\sigma_{t}^{\prime} \gamma_{t}$ if

$$
\sigma_{t}^{\prime i j}=\left(\rho_{t}^{i}\right)^{1 / \alpha} \sigma_{t}^{i j} \quad \text { for } 1 \leq i \leq d, 1 \leq j \leq m .
$$

We thus have (1.2) with $r=m$.

c) Conversely, suppose that (1.2) holds for some $r$ and $Z^{\prime}$ is an i.i.d. symmetric $\alpha$-stable Lévy motion. We will prove that necessarily $m \leq r$.

The Lévy measure of each component of $Z$ (resp. $\left.Z^{\prime}\right)$ is $\nu(d x)=\left(1 / 2 C_{\alpha}|x|^{1+\alpha}\right) d x$, and those components have no common jumps. Hence, since $X=\sigma \cdot Z=\sigma^{\prime} \cdot Z^{\prime}$, the spot Lévy measure $F_{t}^{X}$ of $X$ is, outside a $\overline{\mathbb{P}}$-null set:

$$
F_{t}^{X}(B)=\sum_{j=1}^{q} \int_{\mathbb{R}} 1_{B}\left(x \sigma_{t}^{\cdot, j}\right) \frac{1}{C_{\alpha}|x|^{1+\lambda}} d x=\sum_{j=1}^{r} \int_{\mathbb{R}} 1_{B}\left(x \sigma_{t}^{\cdot, j}\right) \frac{1}{C_{\alpha}|x|^{1+\lambda}} d x .
$$

If for $y \in \mathbb{R}^{d}$ we denote by $D(y)$ the one-dimensional linear subspace of $\mathbb{R}^{d}$ spanned by $y$ (with the convention $D(0)=\{0\})$, (5.10) implies that the support of $F_{t}^{X}$ is $D_{t}=\cup_{1 \leq j \leq q} D\left(\sigma_{t}^{\cdot, j}\right)$ and also $D_{t}^{\prime}=\cup_{1 \leq j \leq r} D\left(\sigma_{t}^{\prime, j}\right)$. Having $D_{t}=D_{t}^{\prime}$ yields that all $\sigma^{\cdot, j}$ for $j=1, \ldots, q$ are proportional to at most $r$ vectors of $\mathbb{R}^{d}$, hence necessarily $m_{t} \leq r$ on the $\overline{\mathbb{P}}$-full set on which (5.10) holds, yielding the claim.

If we now relax the additional condition that in (1.2) the process $Z^{\prime}$ is symmetric i.i.d. $\alpha$-stable, we do no have a general characterization of the minimal $r$ for which this holds. However, this minimal $r$ can still be equal to $q$ when $d \geq 2$ (since when $d=1$ this number is always $r=1$ ).

Theorem 5.2 Let $Z$ be q-dimensional symmetric i.i.d. $\alpha$-stable and $d \geq 2$. One can find a d-dimensional processes $X=\sigma \cdot Z$ for which (1.2) with $Z^{\prime} \in S S S_{r}^{\alpha}$ holds for $r \geq q$ only.

Proof. By looking at the first two components of $X$ it is enough to prove it when $d=2$, and of course when $q \geq 2$.

We will indeed exhibit an $\mathcal{M}_{2, q}$-valued process $\left(\sigma_{t}\right)$ which does not satisfy (1.2) for $r=q-1$, and with a very simple structure. We choose $q$ pairwise non-collinear vectors $w_{j}$ in $\mathbb{R}^{2} \backslash\{0\}$ and use the notation $D\left(w_{j}\right)$ of the previous proof, and let $D=\cup_{j=1}^{q-1} D\left(w_{j}\right)$ and $D^{\prime}=D \cup D\left(w_{q}\right)$. The process $\sigma_{t}$ is defined column-wise by

$$
\sigma_{t}^{\cdot, j}= \begin{cases}w_{j} & \text { if } 1 \leq j \leq q-1 \\ w_{q} 1_{\{t>1\}} & \text { if } j=q\end{cases}
$$

and we also set $D_{t}=D$ if $t \leq 1$ and $D_{t}=D^{\prime}$ if $t>1$.

The rank of $\sigma_{t}$ is 2 for all $t$ and the process $X=\sigma \cdot Z$ is of course well defined. Suppose now that we can find $Z^{\prime}$ in $S S S_{q-1}^{\alpha}$ and a predictable $\mathcal{M}_{2, q-1}$-valued process $\sigma_{t}^{\prime}$ such that $X=\sigma^{\prime} \cdot Z^{\prime}$. If $F^{\prime}$ is the Lévy measure of $Z^{\prime}$, a version of the spot Lévy measure of $X$ is (instead of (5.10)):

$$
F_{t}^{X}(B)=\int_{\mathbb{R}^{q-1}} 1_{B}\left(\sigma_{t}^{\prime} x\right) F^{\prime}(d x)= \begin{cases}\sum_{j=1}^{q-1} \int_{\mathbb{R}} 1_{B}\left(x w_{j}\right) \frac{1}{C_{\alpha}|x|^{1+\lambda}} d x & \text { if } t \leq 1 \\ \sum_{j=1}^{q} \int_{\mathbb{R}} 1_{B}\left(x w_{j}\right) \frac{1}{C_{\alpha}|x|^{1+\lambda}} d x & \text { if } t>1\end{cases}
$$


Therefore, on the one hand, the support of $F_{t}^{X}$ is the image $S_{t}$ of $F^{\prime}$ by the map $x \mapsto f_{t}(x)=$ $\sigma_{t}^{\prime} x$ from $\mathbb{R}^{q-1}$ into $\mathbb{R}^{2}$ and, on the other hand, it is $D_{t}$.

Suppose first $q=2$. Then $\sigma_{t}^{\prime} \in \mathbb{R}^{2}$ and $S_{t}$ is contained in $D\left(\sigma_{t}^{\prime}\right)$, which when $t>1$ contradicts the fact that $S_{t}=D^{\prime}$ contains two non collinear vectors: so $Z^{\prime}$ as above cannot exist.

Suppose now $q \geq 3$. The linear space spanned by $S_{t}=D_{t}$ is of dimension 2 for all $t$, so the matrix $\sigma_{t}^{\prime}$ has rank 2 and thus $f_{t}$ is a bijection from a two-dimensional subspace $E_{t}$ of $\mathbb{R}^{q-1}$ into $\mathbb{R}^{2}$, and the lines $\bar{D}_{t}^{j}=f_{t}^{-1}\left(D\left(w_{j}\right)\right)$ in $E_{t}$ are pairwise distinct because the lines $D\left(w_{j}\right)$ are such in $\mathbb{R}^{2}$, whereas $\bar{D}_{t}=f_{t}^{-1}\left(D_{t}\right)$ is $\cup_{j=1}^{q-1} \bar{D}_{t}^{j}$ if $t \leq 1$ and $\cup_{j=1}^{q} \bar{D}_{t}^{j}$ it $t>1$. Moreover, $S_{t}=D_{t}$ implies that $F^{\prime}\left(\mathbb{R}^{q-1} \backslash \bar{D}_{t}\right)=0$ and $F^{\prime}$ puts a positive mass on all lines $\bar{D}_{t}^{j}$ in $\bar{D}_{t}$. Since there are $q-1$ such distinct lines when $t \geq 1$ and $q$ of them when $t>1$, this is clearly impossible. So again $Z^{\prime}$ as above cannot exist.

\section{References}

[1] O. Bardou (2005): Contrôle dynamique des erreurs de simulation et d'estimation de processus de diffusion. Ph.D. thesis, Univ. de Nice Sophia Antipolis.

[2] D. Brigo and F. Mercurio (2006): Interest rates models-theory and practice. Springer Finance, 2nd Edition.

[3] T. Fissler and M. Podolskij (2016): Testing the maximal rank of the volatility process for continuous diffusions observed with noise. To appear in Bernoulli.

[4] J. Jacod, A. Lejay and D. Talay (2008): Estimation of the Brownian dimension of a continuous Itô process. Bernoulli 14, 469-498.

[5] J. Jacod and M. Podolskij (2013): A test for the rank of the volatility process: The random perturbation approach. Annals of Statistics 41(5), 2391-2427.

[6] K.-I. Sato (1999): Lévy processes and infinitely divisible distributions. Cambridge University Press. 